\title{
Tunneling Conductance in Ferromagnetic Metal/Normal Metal/Spin-Singlet $s$-Wave Ferromagnetic Superconductor Junctions
}

\author{
Hamidreza Emamipour \\ Department of Physics, Ilam University, Ilam, Iran \\ Correspondence should be addressed to Hamidreza Emamipour; h_emamipour@yahoo.com
}

Received 2 May 2013; Revised 2 August 2013; Accepted 24 September 2013

Academic Editor: A. Bansil

Copyright (C) 2013 Hamidreza Emamipour. This is an open access article distributed under the Creative Commons Attribution License, which permits unrestricted use, distribution, and reproduction in any medium, provided the original work is properly cited.

In the framework of scattering theory, we study the tunneling conductance in a system including two junctions, ferromagnetic metal/normal metal/ferromagnetic superconductor, where ferromagnetic superconductor is in spin-singlet $s$-wave pairing state. The non-magnetic normal metal is placed in the intermediate layer with the thickness $(L)$ which varies from $1 \mathrm{~nm}$ to $10000 \mathrm{~nm}$. The interesting result which we have found is the existence of oscillations in conductance curves. The period of oscillations is independent of FS and FN exchange field while it depends on $L$. The obtained results can serve as a useful tool to determine the kind of pairing symmetry in ferromagnetic superconductors.

\section{Introduction}

In this paper we have studied tunneling conductance in a two-junction system with structure of ferromagnetic metal/normal metal/ferromagnetic superconductor. A constant spin-singlet $s$-wave pairing potential $\Delta$ and a constant exchange field $h_{s}$ are our presuppositions for the ferromagnetic superconductor. The main goal of this paper is to show the existence of oscillations in conductance curves with period of oscillations independent of FS and FN exchange field but dependent on $L$. The structure of this paper is as follows. In Section 1 a historical view of the under study subject together with a short report of the results is presented. In Section 2 the model and the theoretical framework of our system are studied. In Section 3 we have presented and discussed the results of the paper.

For a long time, coexistence of superconductivity and ferromagnetism has been a challenging issue in the history of superconductivity. It was believed that superconductivity and ferromagnetism were two opposite phenomena and coexistence between them was impossible. The view toward ferromagnetism and superconductivity changed when ferromagnetic superconductors were discovered in 2000-2001.
The discovery of superconductivity in some ferromagnetic materials such as $U G e_{2}$ [1], $Z r Z n_{2}$ [2], and $U R h G e$ [3] has attracted much attention in the field of superconductivity.

The questions are mostly raised about the mechanism of cooper pairing in ferromagnetic superconductors. At the first glance, it seems that the pairing symmetry of such materials should be spin triplet equal spin pairing. But some research groups have shown that one could not ignore spin-singlet $s$ wave opposite spin pairing [4-11]. For example, in [7], Singh and Mazin by calculating the electronic structure, as well as the zone center phonons and their coupling with electrons, have shown that $Z r Z n_{2}$ has a spin-singlet $s$-wave pairing state. Also, in [8], due to kinetic exchange, the coexistence between ferromagnetism and $s$-wave pairing has been shown. Also, Dahal et al. [9], based on interaction of electrons mediated by localized spins have shown that the pair symmetry in $U G e_{2}$ is spin-singlet $s$-wave state. (Thus, in this paper, we consider spin-singlet $s$-wave pairing for ferromagnetic superconductors.) Other pairing symmetries such as spin triplet opposite spin pairing (OSP) state and spin triplet equal spin pairing (ESP) state are suggested for ferromagnetic superconductors $[12,13]$. These two pairing states are different from SWP state, because their order parameters have a sign 
change on Fermi surface, which leads to the formation of a midgap state while there is not midgap state in the case of SWP state. The formation of midgap states affects the tunneling spectra, so they make difference between different symmetries in terms of the kind of pair symmetries [12].

On the other hand, tunneling spectroscopy can provide much information about the excitation energy, the type of pairing symmetry, and other physical properties of superconductors. For the first time, the theory of tunneling spectroscopy by taking into account Andreev reflection [14] was presented by Blonder et al. (BTK theory) in 1982 [15]. After the discovery of high temperature superconductors, Tanaka and Kashiwaya have extended BTK theory to unconventional $d$-wave superconductors in $1995[16,17]$. The presence of zero-bias conductance peaks in tunneling spectroscopy of normal metal/unconventional superconductor junctions is accepted as a strong indication of $d$-wave symmetry in high temperature superconductors.

In order to determine the type of pairing symmetry in ferromagnetic superconductors, the detailed comparison between theoretical results and experimental data is required. Anyway, as it is mentioned in [18], the study of tunneling conductance in junctions which consist of ferromagnetic superconductors can settle the problem. Till now, the study of tunneling spectroscopy for ferromagnetic superconductors has been restricted to one junction (see $[12,13,19,20]$ ).

In this paper, we theoretically study tunneling conductance in ferromagnetic metal/normal metal/ferromagnetic superconductor (FM/NM/FSc) double junction where ferromagnetic superconductor (FS) is in spin-singlet $s$-wave pairing (SWP) state and has an exchange field $h_{s}$. The nonmagnetic normal metal is placed in an intermediate layer with a zero barrier potential and the thickness of $L$ which varies from $1 \mathrm{~nm}$ to $10000 \mathrm{~nm}$. We find that the tunneling conductance oscillates as a function of bias voltage. The period of oscillations is independent of $h_{s}$ and $h_{f}$ (exchange field of ferromagnetic metal), while it is inversely proportional to $L$. From the obtained results, we can obtain much information about the symmetry of pair potential of ferromagnetic superconductors which is a controversial issue in the field of superconductivity.

\section{Formulation}

In Figure 1 we have shown the structure of the system which we study in the current paper. Figure 1(a) shows a schematic diagram of FM/NM/FS double junctions. An NM with the thickness of $L$ is sandwiched by FM and FS layers. Figure 1(b) shows a schematic diagram of energy versus wave vector at FM, NM, and FS regions, respectively. The open circles, the filled circles, and the arrows point denote holes, electrons, and the direction of the group velocity, respectively. The incident electron is indicated by 0 and experiences the following processes: (1) Andreev reflection, (2) normal reflection, (3, 4) transmission to the NM, $(5,6)$ reflections at the NM/FS interface, and $(7,8)$ transmission to the FS as electron and hole quasiparticles. We study a system consisting of two junctions, ferromagnetic metal/normal metal/ferromagnetic superconductor (FM/NM/FSc); that is, one junction is placed between a ferromagnetic metal and a normal metal, and the other one is between a normal metal and a ferromagnetic superconductor. The junction NM/FSc is located at $x=L$. The nonmagnetic normal metal is placed between two junctions with the thickness of $L$, and ferromagnetic metal (FM) and the ferromagnetic superconductor (FSc) are located at $x<0$ and $x>L$, respectively. The exchange field of the system is in the following form:

$$
h(x)=h_{f}(1-\Theta(x))+h_{s} \Theta(x-L),
$$

where $h_{f}$ and $h_{s}$ are the exchange fields of FM and FSc, respectively, and $\Theta(t)$ is the step function which is one for $t>0$ and zero for $t<0$. The effective pair potential for FSc is defined by $\Delta(\vec{k}, \vec{r})=\Delta(\vec{k}) \Theta(x-L)$, where for spin-singlet $s$ wave pairing $\Delta(\vec{k})$ is in the following form: $\Delta(\vec{k})=i \Delta \sigma_{y}[12]$. The motion of quasiparticles is described by Bogoliubov-De Gennes (BdG) equations which have the following form:

$$
\begin{aligned}
& {\left[H_{0}-\vec{h} \cdot \vec{\sigma}\right] f(\vec{r})+\Delta(\vec{r}) g(\vec{r})=E f(\vec{r})} \\
& -\left[H_{0}+\vec{h} \cdot \vec{\sigma}\right] g(\vec{r})+\Delta(\vec{r}) f(\vec{r})=\operatorname{Eg}(\vec{r}),
\end{aligned}
$$

where $H_{0}=-\left(\hbar^{2} / 2 m\right) \nabla^{2}-E_{F}$ is the one particle Hamiltonian. $E$ is the energy of quasi-particles which is measured from Fermi energy; $E_{F} . \vec{\sigma}\left(=\sigma_{x} \widehat{x}+\sigma_{y} \widehat{y}+\sigma_{z} \widehat{z}\right)$ is the Pauli matrices where $\hat{x}, \widehat{y}$, and $\widehat{z}$ are the unit vectors in the $x$-, $y$-, and $z$ directions, respectively. $\vec{h}$ is the exchange field of system; we assume that it is in the $z$-direction; $\vec{h}=\widehat{z} h(x)$ and $h(x)$ is defined by (1).

Now, we are ready to find the eigenfunction and eigenvalues from (2). By replacing (1) in (2) and solving it, the energy spectrum for an electron is obtained from the following formula:

$$
E_{ \pm}(k)=\sqrt{\varepsilon_{k}^{2}+|\Delta|^{2}} \pm h_{s}
$$

and the energy spectrum of a hole is: $E=-E_{ \pm}$, where $E_{ \pm}$is defined in (3).

The corresponding wave functions of spin-singlet $s$-wave state are obtained in the following forms [13]:

$$
\begin{array}{ll}
u_{-\sigma \sigma}=\sqrt{\frac{1}{2}\left(1+\frac{\sqrt{\left(E_{\sigma}-\sigma h_{s}\right)^{2}-|\Delta|^{2}}}{E_{\sigma}-\sigma h_{s}}\right)}, & \sigma= \pm, \\
v_{-\sigma \sigma}=\sqrt{\frac{1}{2}\left(1-\frac{\sqrt{\left(E_{\sigma}+\sigma h_{s}\right)^{2}-|\Delta|^{2}}}{E_{\sigma}+\sigma h_{s}}\right)}, & \sigma= \pm .
\end{array}
$$

So, the wave function in $x>L$ region whose ferromagnetic superconductor (FS) is placed there is as follows:

$$
\Psi_{\mathrm{FS}}(x)=c\left(\begin{array}{l}
u \\
v
\end{array}\right) e^{i k_{\mathrm{FS}}^{+} x}+d\left(\begin{array}{l}
v \\
u
\end{array}\right) e^{-i k_{\mathrm{FS}}^{-} x}
$$

where $k_{\mathrm{FS}}^{ \pm}=k_{F} \sqrt{1 \pm \sqrt{\left(E \pm h_{s}\right)^{2}-\left|\Delta_{o}\right|^{2}} / E_{F}}$ is the wave vector of FS layer. 


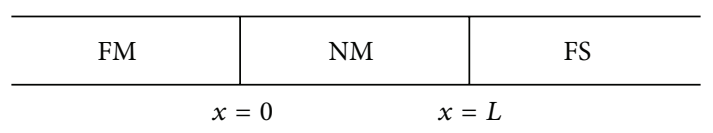

(a)

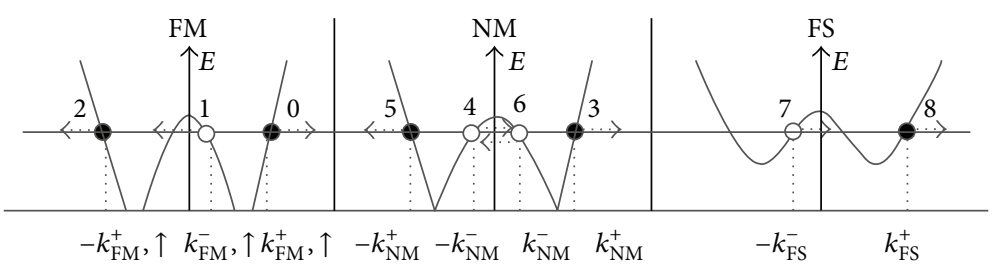

(b)

FIGURE 1: (a) Schematic diagram of a FM/NM/FS double junctions. (b) Schematic diagram of energy versus wave vector of a FM/NM/FS double junctions for a spin-up incident electron.

In intermediate layer $(0<x<L)$ whose normal metal (NM) is placed, the wave function is obtained by setting $\vec{h}=0$ and $\Delta=0$ in (3). In this case the solution of (3) is obtained as follows:

$$
\begin{aligned}
& \Psi_{\mathrm{NM}}(x) \\
& =\alpha_{\sigma}(E)\left(\begin{array}{l}
1 \\
0
\end{array}\right) e^{i k_{\mathrm{NM}}^{+} x}+\beta_{\sigma}(E)\left(\begin{array}{l}
1 \\
0
\end{array}\right) e^{-i k_{\mathrm{NM}}^{+} x} \\
& +\alpha_{\sigma}^{\prime}(E)\left(\begin{array}{l}
0 \\
1
\end{array}\right) e^{-i k_{\mathrm{NM}}^{-} x}+\beta_{\sigma}^{\prime}(E)\left(\begin{array}{l}
0 \\
1
\end{array}\right) e^{i k_{\mathrm{NM}}^{-}},
\end{aligned}
$$

where $k_{\mathrm{NM}}^{ \pm}=k_{F} \sqrt{1 \pm\left(E / E_{F}\right)}$ is the wave vector of $\mathrm{NM}$ region.

In the $x<0$ region where ferromagnetic metal (FM) is placed, the wave function is as follows:

$$
\begin{aligned}
\Psi_{\mathrm{FM}}(x)= & \left(\begin{array}{l}
1 \\
0
\end{array}\right) e^{i K_{\mathrm{FM}}^{+} x} \\
& +a_{\sigma}(E)\left(\begin{array}{l}
0 \\
1
\end{array}\right) e^{i K_{\mathrm{FM}}^{-} x}+b_{\sigma}(E)\left(\begin{array}{l}
1 \\
0
\end{array}\right) e^{-i K_{\mathrm{FM}}^{+} x},
\end{aligned}
$$

where $K_{\mathrm{FM}}^{ \pm}=k_{F} \sqrt{1 \pm\left(h_{f} / E_{F}\right) \pm\left(E / E_{F}\right)}$ is the wave vector in FM region and $a$ and $b$ are the Andreev and normal coefficients, respectively.

Now, according to BTK theory, we should replace the above wave functions in the following boundary conditions:

$$
\begin{aligned}
& \psi_{\mathrm{FM}}(x=0)=\psi_{\mathrm{NM}}(x=0), \\
& \psi_{\mathrm{NM}}(x=L)=\psi_{\mathrm{FS}}(x=L), \\
& \psi_{\mathrm{FM}}^{\prime}(x=0)=\psi_{\mathrm{NM}}^{\prime}(x=0), \\
& \psi_{\mathrm{NM}}^{\prime}(x=L)=\psi_{\mathrm{FS}}^{\prime}(x=L),
\end{aligned}
$$

where $\psi_{\mathrm{FM}}, \psi_{\mathrm{NM}}$, and $\psi_{\mathrm{FS}}$ are the wave functions of FM, $\mathrm{NM}$, and FSc; respectively, and $\psi^{\prime}$ is the derivative of $\psi$ with respect to $x$; that is, $\psi^{\prime}=d \psi / d x$. By applying the above boundary conditions, we have obtained Andreev and normal coefficients, and as the result, we have obtained the conductance $\left(G_{\sigma}=1+\left|a_{\sigma}\right|^{2}-\left|b_{\sigma}\right|^{2}\right)$ in the following form:

$$
G_{\sigma}=\frac{32\left|v_{1} / u_{1}\right|^{2} k^{2} q q^{\prime}}{\left|(k+q)\left(k+q^{\prime}\right)-\left(v_{1} v_{2} / u_{1} u_{2}\right)(k-q)\left(k-q^{\prime}\right) e^{i\left(k-k^{\prime}\right) L}\right|^{2}},
$$

where we have

$$
\begin{array}{ll}
k=k_{F} \sqrt{1+\frac{E}{E_{F}}}, & k^{\prime}=k_{F} \sqrt{1-\frac{E}{E_{F}}}, \\
q=k_{F} \sqrt{1+\frac{h_{f}}{E_{F}}}, & q^{\prime}=k_{F} \sqrt{1-\frac{h_{f}}{E_{F}}} .
\end{array}
$$

The normalized tunneling conductance is obtained from this formula: $G=\left(\sum_{\sigma} G_{\sigma}\right) / G_{\mathrm{N}}$, where $G_{\mathrm{N}}$ is the conductance of $\mathrm{N} / \mathrm{N} / \mathrm{N}$ junction.

Before we present the results, to examine the validity of our calculation, we compare (10) with previously done researches. In [13], we have calculated zero-bias conductance for NM/FSc junction. By placing $z=0$ in results of [13] and $L=0$ and in (10), we come up with the same result. Also, in [21], the tunneling conductance is calculated for FM/Sc junction. In the limit of $L \rightarrow 0$, the results of [21] are reproduced. Also, in the limit of $h_{s} \rightarrow 0$ the results of [22] are reproduced which shows the validity of our calculations.

\section{Results}

Figure 2 shows the conductance curves for $L=1000 \mathrm{~nm}$ and $h_{s} / \Delta=0.2,0.4,0.6$. As it can be seen in Figure 2(a), the conductance shows a periodic behavior especially in subgap region $(E<\Delta)$. The origin of this periodic behavior comes back to the interference of electron waves in the intermediate layer $[23,24]$. We can interpret this periodic behavior by using the energy diagram of Figure 1(b). In fact the incident electron propagates through the NM layer toward the NM/FS interface as an electron (3 in Figure 1(b)) and is diffused into a hole (6 in Figure 1(b)) by the FS order parameter. The FS order parameter can couple an excited electron with an electron inside the Fermi sea, and it leaves a hole excitation. This hole is scattered back into the NM layer, but it does not interfere with the primary electron. In order to take place an interference at the NM layer, the hole should be reflected at the NM/FS interface, be propagated into the electron state (5 in Figure 1(b)) by the FS order parameter, and then propagate into the NM layer as an electron. So, the interference at the NM layer can occur with the primary electron (3 in Figure 1(b)), and it produces an oscillation in the conductance curves at Figure 2(a). The effect of midgap states on conductance curves is an interesting subject. For example, in the case of SWP state there is not midgap state at the interface. So, we 


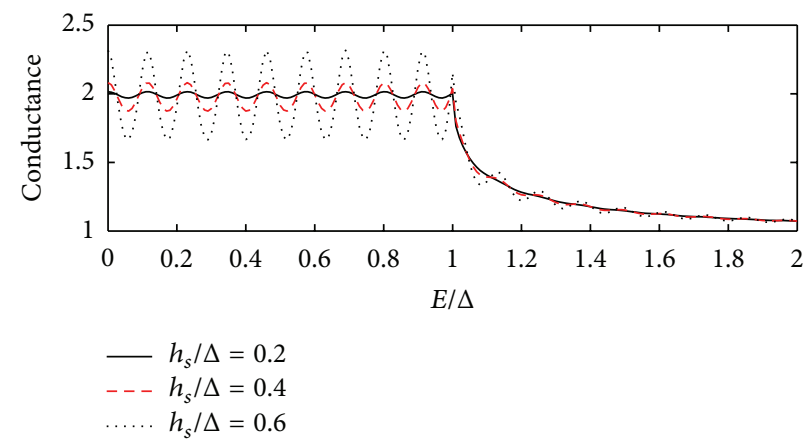

(a)

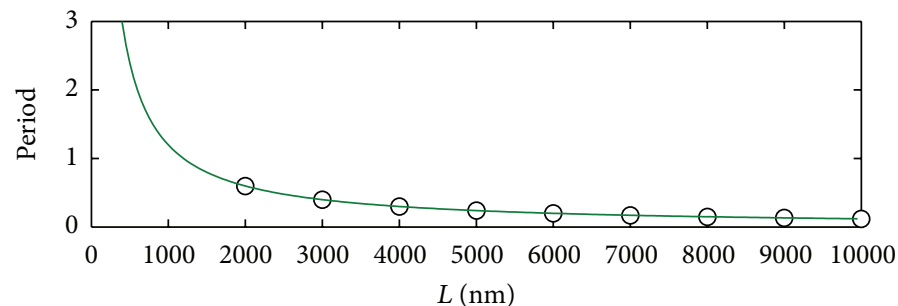

(b)

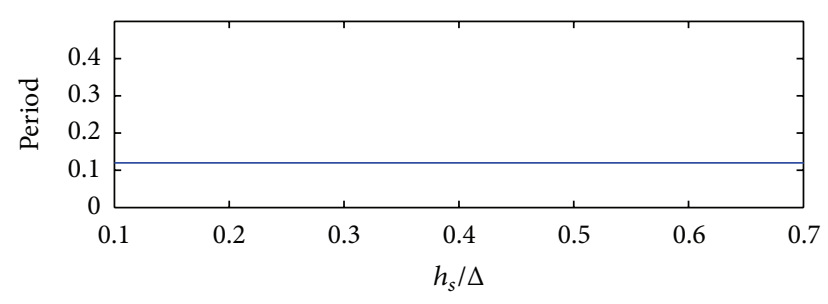

(c)

FIgURE 2: (a) Normalized tunneling conductance versus bias voltage for different exchange fields, (b) period of conductance oscillations versus thickness of intermediate layer, and (c) period of conductance oscillations versus exchange field of ferromagnetic superconductor.

have not zero-bias conductance peak (ZBCP), while as it is mentioned in $[12,13]$ ZBCP exists for the case of OSP and ESP states.

Also, we can see with the increase in exchange field, the magnitude of conductance decreases. This is because with the increase in exchange field, the symmetry of spin-singlet $s$ wave pairing breaks and so conductance decreases. In Figure 2(a), we can see that in $\Delta \ll E$, normalized conductance tends to 1 . It is because when $|\Delta|$ is small in comparison with the energy of bias voltage, the FS tends to NM, and so normalized conductance which is defined by $\left(G=\left(\sum_{\sigma} G_{\sigma}\right) / G_{N}=\right.$ $\left.G_{\mathrm{F} / \mathrm{N} / \mathrm{FS}} / G_{\mathrm{F} / \mathrm{N} / \mathrm{N}}\right)$ tends to 1 .

In Figures 2(b) and 2(c), we study the period of conductance oscillation. Analytically by replacing $\left(k-k^{\prime}\right) L=\pi$ in (9), we find the following equation for the period of oscillations:

$$
\text { period }=\frac{381 \pi}{L(\mathrm{~nm})},
$$

which is obtained for $k_{F}=10 \mathrm{~nm}^{-1}$. We note that (11) may change when we have a two- dimensional junction with other symmetries. For example, in the case of a $d$-wave symmetry, the interferences take place at two directions, and the period of oscillations may be obtained from $\left(k-k^{\prime}\right) L \cos \theta=\pi$. Therefore, (11) has a different form in the case of other symmetries. We examine (11) numerically. We measure the period of oscillations from Figure 2(a) and we find a good agreement between numerical data and analytic result (see (11)). Solid line in Figure 2(b) is plotted from (11) and circles in Figure 2(b) are obtained from numerical data. As it can be seen from this figure there is good correspondence between numerical and analytical results.
Figure 2(c) shows that the period of oscillations does not depend on the FS exchange field. We have plotted different $G-E$ curves for different exchange fields and we have found that the period of oscillations is independent of exchange field. This is because the period of oscillations just depends on $k$ and $k^{\prime}$ while it does not depend on exchange fields. A point that should be noted is that Figure 2(c) is plotted for the values of $h_{s}<0.7 \Delta$. This is because at zero temperature for the values of $h_{s}>0.7 \Delta$ ferromagnetic superconducting state converts to normal metal state. In fact, as it is mentioned in $[13,25]$, by calculating the difference in thermodynamic potential between superconducting state and normal state, $\Omega_{s}-\Omega_{n}$, we see that for $h_{s}>0.7 \Delta$ the thermodynamic potential of normal metal is lower than the thermodynamic potential of superconducting state, $\Omega_{n}<\Omega_{s}$. So in a first order phase transition process, the superconducting state falls into normal state, and in the presence of an exchange field, superconducting state exists just for $h_{s}<0.7 \Delta$.

Beside spin-singlet $s$-wave pairing (SWP) state, the other symmetries such as spin triplet opposite spin pairing (OSP) state and spin triplet equal spin pairing (ESP) state are suggested for ferromagnetic superconductors $[12,13]$. As it is mentioned at $[12,13]$, one of the characteristic features of OSP and ESP states is the existence of zero-bias conductance peaks in conductance curves. This comes back to the type of their pair potentials. In OSP and ESP states the sign change of pair potential at Fermi surface leads to the formation of midgap Andreev resonant states, and they are responsible for the formation of zero bias conductance peaks. There is not conductance peak at zero-bias for SWP state, because its pair potential does not change at the Fermi surface. In fact the kind of pair potential strongly affects the tunneling 
conductance and so our results can help distinguish the type of symmetry in ferromagnetic superconductors which is still unknown for physicists.

\section{References}

[1] S. S. Saxena, P. Agarwal, K. Ahilan et al., "Superconductivity on the border of itinerant-electron ferromagnetism in UGe2," Nature, vol. 406, no. 6796, pp. 587-592, 2000.

[2] C. Pfleiderer, M. Uhlarz, S. M. Hayden et al., "Coexistence of superconductivity and ferromagnetism in the d-band metal ZrZn2," Nature, vol. 412, no. 6842, pp. 58-61, 2001.

[3] D. Aoki, A. Huxley, E. Ressouche et al., "Coexistence of superconductivity and ferromagnetism in URhRe," Nature, vol. 413, no. 6856, pp. 613-616, 2001.

[4] A. A. Abrikosov, "Superconductivity due to ferromagnetically ordered localized spins," Journal of Physics Condensed Matter, vol. 13, no. 48, pp. L943-L947, 2001.

[5] H. Suhl, "Simultaneous onset of ferromagnetism and superconductivity," Physical Review Letters, vol. 87, no. 16, Article ID 167007, 3 pages, 2001.

[6] N. I. Karchev, K. B. Blagoev, K. S. Bedell, and P. B. Littlewood, "Coexistence of superconductivity and ferromagnetism in ferromagnetic metals," Physical Review Letters, vol. 86, no. 5, pp. 846-849, 2001.

[7] D. J. Singh and I. I. Mazin, "Competition of spin fluctuations and phonons in superconductivity of ZrZn2," Physical Review Letters, vol. 88, no. 18, Article ID 1870044, 2002.

[8] M. Cuoco, P. Gentile, and C. Noce, "Coexistence of ferromagnetism and singlet superconductivity via kinetic exchange," Physical Review Letters, vol. 91, no. 19, Article ID 197003, 4 pages, 2003.

[9] H. P. Dahal, J. Jackiewicz, and K. S. Bedell, "Pairing symmetry signatures of T1 in superconducting ferromagnets," Physical Review B, vol. 72, no. 17, Article ID 172506, 2005.

[10] H. Kotegawa, A. Harada, S. Kawasaki et al., "Evidence for uniform coexistence of ferromagnetism and unconventional superconductivity in UGe2: a 73Ge-NQR study under pressure," Journal of the Physical Society of Japan, vol. 74, no. 2, pp. 705-711, 2005.

[11] K. B. Blagoev, J. R. Engelbrecht, and K. S. Bedell, "S-wave superconductivity in weak ferromagnetic metals," Philosophical Magazine Letters, vol. 78, no. 2, pp. 169-176, 1998.

[12] T. Yokoyama and Y. Tanaka, "Theory of the tunneling spectroscopy of ferromagnetic superconductors," Physical Review B, vol. 75, Article ID 132503, 4 pages, 2007.

[13] H. Emamipour and M. R. Abolhasani, "Temperature dependence of zero-bias conductance in a normal metal/ferromagnetic superconductor junction," Superconductor Science and Technology, vol. 23, no. 10, Article ID 105001, 2010.

[14] A. F. Andreev, "Thermal conductivity of the intermediate state of superconductors," Soviet Physics: Journal of Experimental and Theoretical Physics, vol. 19, pp. 1228-1231, 1964.

[15] G. E. Blonder, M. Tinkham, and T. M. Klapwijk, "Transition from metallic to tunneling regimes in superconducting microconstrictions: excess current, charge imbalance, and supercurrent conversion," Physical Review B, vol. 25, no. 7, pp. 4515-4532, 1982.

[16] Y. Tanaka and S. Kashiwaya, "Theory of tunneling spectroscopy of d-wave superconductors," Physical Review Letters, vol. 74, no. 17, pp. 3451-3454, 1995.
[17] S. Kashiwaya, Y. Tanaka, M. Koyanagi, and K. Kajimura, “Theory for tunneling spectroscopy of anisotropic superconductors," Physical Review B, vol. 53, no. 5, pp. 2667-2676, 1996.

[18] Y. Asano, Y. Tanaka, A. A. Golubov, and S. Kashiwaya, "Conductance spectroscopy of spin-triplet superconductors," Physical Review Letters, vol. 99, Article ID 067005, 4 pages, 2007.

[19] H. Emamipour and M. R. Abolhassanit, "Tunneling conductance in a normal metal/ferromagnetic superconductor nanojunction at a finite temperature," Communications in Theoretical Physics, vol. 55, no. 1, pp. 171-175, 2011.

[20] H. Emamipour and J. Emamipour, "Zero-bias conductance versus potential strength of interface in ferromagnetic superconductors," Chinese Physics Letters, vol. 29, no. 3, Article ID 037401, 2012.

[21] M. J. M. de Jong and C. W. J. Beenakker, "Andreev reflection in ferromagnet-superconductor junctions," Physical Review Letters, vol. 74, no. 9, pp. 1657-1660, 1995.

[22] H. Ohtori and H. Imamura, "Conductance oscillations due to geometrical resonance in FNS double junctions," Physical Review B, vol. 79, Article ID 212507, 4 pages, 2009.

[23] P. G. de Gennes and D. Saint-James, "Elementary excitations in the vicinity of a normal metal-superconducting metal contact," Physics Letters, vol. 4, no. 2, pp. 151-152, 1963.

[24] J. M. Rowell and W. L. McMillan, "Electron interference in a normal metal induced by superconducting contracts," Physical Review Letters, vol. 16, no. 11, pp. 453-456, 1966.

[25] X. Li, Z. Zheng, D. Y. Xing, G. Sun, and Z. Dong, "Josephson current in ferromagnet-superconductor tunnel junctions," Physical Review B, vol. 65, Article ID 134507, 6 pages, 2002. 

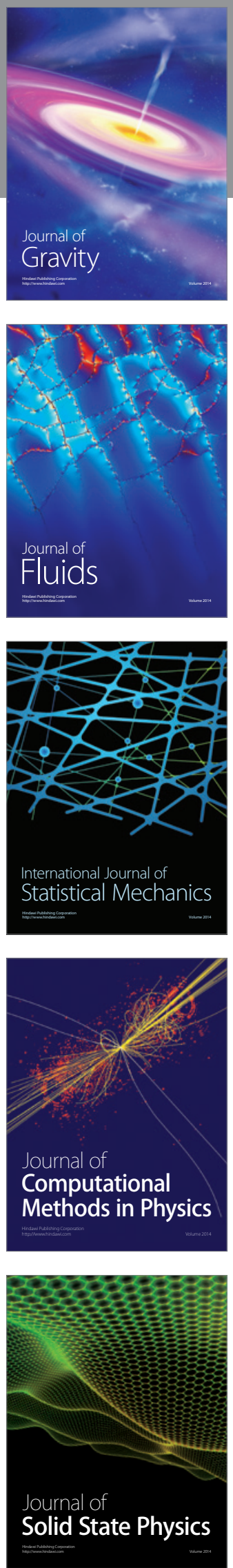

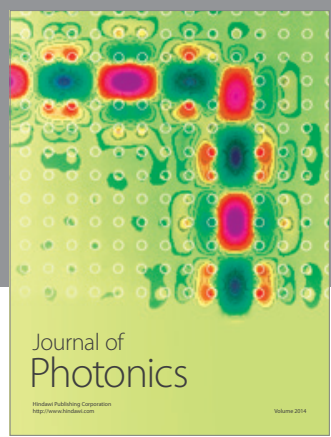

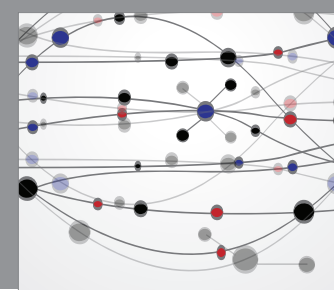

The Scientific World Journal

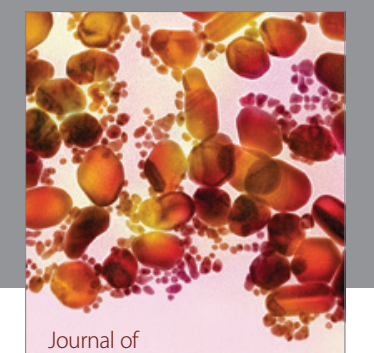

Soft Matter
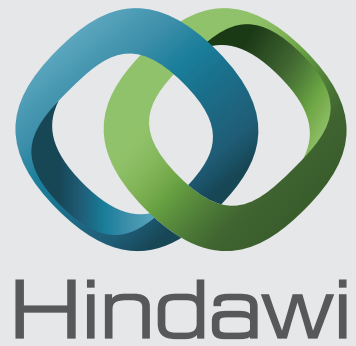

Submit your manuscripts at

http://www.hindawi.com
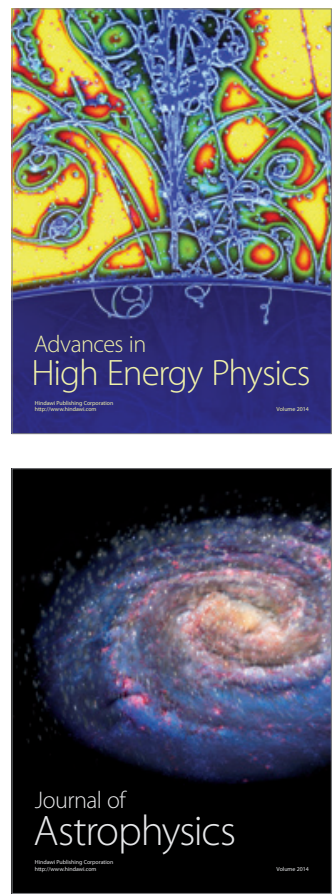
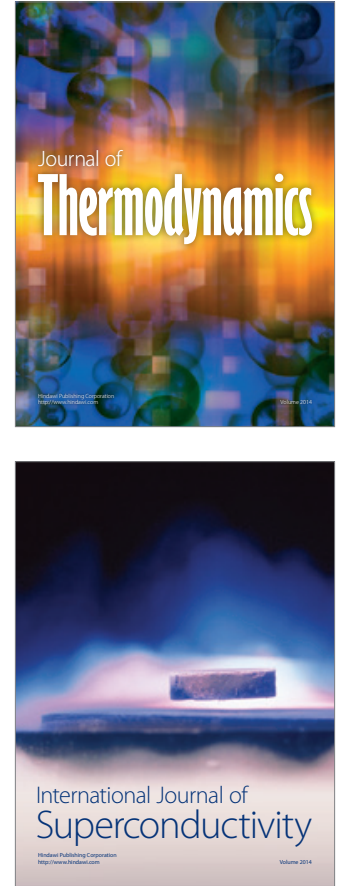
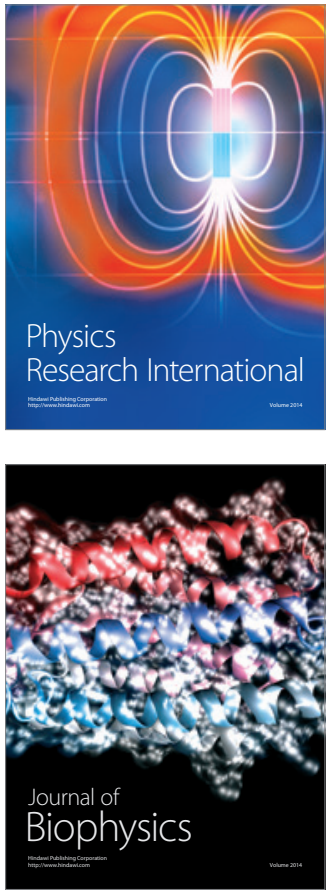
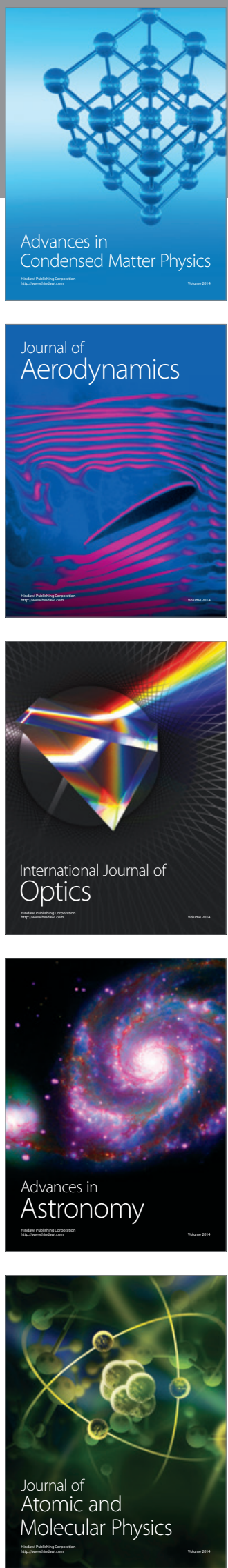